\title{
Study on Horizontally Polarized Omnidirectional Microstrip Antenna
}

\author{
Kun Wei, Jian-ying Li, Ling Wang, Zi-jian Xing, and Rui Xu \\ School of Electronics and Information, Northwestern Polytechnical University, Xian 710072, China \\ Correspondence should be addressed to Kun Wei; weikun916@163.com
}

Received 27 October 2015; Revised 11 December 2015; Accepted 17 December 2015

Academic Editor: Giuseppe Mazzarella

Copyright (C) 2016 Kun Wei et al. This is an open access article distributed under the Creative Commons Attribution License, which permits unrestricted use, distribution, and reproduction in any medium, provided the original work is properly cited.

\begin{abstract}
A horizontally polarized omnidirectional microstrip antenna is proposed in this paper. The structure of designed antenna is with two back-to-back horizontally polarized microstrip antenna elements. Gain variation on main radiation plane of this new antenna is analyzed and radiation theory is deduced; formula of directivity on main radiation plane is given. Better omnidirectional characteristic of this antenna can be obtained by decreasing patch physical length. Both simulated and measured results verify the omnidirectional radiation patterns and input impedance characteristics. Good omnidirectional radiation patterns (gain variation in $E$-plane less than $\pm 0.4 \mathrm{dBi}$ ) and input impedance characteristics are obtained; moreover, cross polarization less than $-20 \mathrm{dBi}$ is achieved.
\end{abstract}

\section{Introduction}

Omnidirectional antenna has been widely used in mobile communication, indoor radio, and wireless LAN systems because of their better performance in multiple paths environment. It is well known that an omnidirectional antenna can be easily realized by a vertical dipole [1-4]. However, vertical dipole works in vertical polarization mode rather than horizontal polarization mode.

Microstrip antennas are also designed with omnidirectional radiation pattern. Microstrip antenna array mounted on a circular cylinder is presented in [5]; it needs more than eleven patches to achieve good omnidirectional pattern. The drawbacks of those cylindrical conformal microstrip antennas are difficult to fabricate and they need more than three patches to obtain omnidirectional radiation pattern. Back-to-back omnidirectional microstrip antennas in [69] are with omnidirectional radiation patterns; however, those antennas are either vertically polarized or circularly polarized; none of the antennas in [5-9] are working in horizontal polarization mode.

Horizontally polarized (HP) omnidirectional antennas can be realized by printed antenna and slot antenna. In [10], there are four notches cut out from the bottom conductor layer, and four microstrip lines feed them, respectively, on the upper layer to obtain HP omnidirectional radiation pattern. A horizontally polarized omnidirectional planar antenna in [11] is developed for mobile communications; the proposed antenna consists of four printed arc dipoles that form a circular loop for HP omnidirectional radiation, but antenna size in $[10,11]$ is bigger than that of antenna proposed in this paper. In [12], a HP omnidirectional planar printed antenna for WLAN applications is presented. The printed Alford-loopstructure antenna consists of two Z-shaped strips printed on the top and bottom plane of the FR-4 printed-circuit-board substrate, but average antenna gain (horizontal-polarization field) is only $-5.3 \mathrm{dBi}$.

Slot antenna or array may also obtain omnidirectional radiation pattern [13-15]. A three-element CPW-fed leaky wave folded slot antenna array is presented in [13]. By using the columnar structure, the gain variation in the azimuthal plane is less than $1.1 \mathrm{~dB}$. A planar slot array antenna with omnidirectional radiation pattern in the horizontal plane is proposed in [14]; the antenna with eight back-to-back slots is designed by employing the genetic algorithm implemented on a cluster system to achieve omnidirectional radiation 
characteristics. Drawbacks of slot antennas or arrays obtained horizontally polarized omnidirectional radiation pattern are large in size, heavy in weight, and difficult to fabricate.

A horizontally polarized omnidirectional microstrip antenna is described in [16]; the antenna consists of three main components: a probe-fed main patch and two parasitic patches, placed conformally on a cylindrical structure. The antenna structure is designed in the form of a belt for horizontally polarized applications. But the $-10 \mathrm{~dB}$ antenna bandwidth is about $1.46 \%$ and the antenna is hard to fabricate.

A new horizontally polarized omnidirectional microstrip antenna is proposed in this paper. The designed antenna, which has a pair of patches, is with back-to-back structure. The patches are symmetrically placed along vertical plane, so fields from the two patches of the designed antenna are interfered constructively (add) in the horizontal direction and interfered destructively (cancel each other) in vertical direction. Due to that, omnidirectional radiation pattern is achieved. Adding that patches are horizontally polarized, so designed antenna is with horizontally polarized omnidirectional radiation pattern. Wilkinson power divider is used for feeding the patches, because it is with the useful property of appearing lossless when the output ports are matched. This characteristic provides good impedance matching and good efficiency performances of designed antenna in this paper.

The radiation theory of the back-to-back structure is studied. Software Ansoft HFSS is applied to design and simulate the antenna for the purpose of optimizing the omnidirectional characteristic, drawing the best technical parameters. The new proposed antenna is fabricated and measured. Measured results are shown to make the comparison with the simulated ones. The proposed HP omnidirectional microstrip antenna enjoys the following advantages: (1) it has a small and compact size; (2) it is easy to fabricate; (3) the radiation pattern and input impedance characteristic are satisfactory; (4) the cross polarization level is low.

\section{Antenna Design}

The geometry of designed antenna is shown in Figure 1(a). Outline size of the antenna is as follows: length $b=32 \mathrm{~mm}$, width $a=10 \mathrm{~mm}$, and height $h=65 \mathrm{~mm}$. For better illustration of the designed antenna structure, the model is divided into four parts as shown in Figure 1(b), namely, two external layers and two internal layers. There is no gap between each layer in practical design. Both external layers are singlefed rectangular-patch microstrip antennas with back-to-back structure. And the internal layers constitute a classic striplinetype Wilkinson power divider, where port 1 is input port and port 2 and port 3 are two output ports with the same signal amplitude and phase. Two patches are connected with output ports of the power divider differently by probes, which are inner core of coaxial line with length $5 \mathrm{~mm}$ and radius $0.45 \mathrm{~mm}$. Port 2 is used to excite the right patch shown in Figure 1(b) and port 3 feeds the left patch. Figure 1(c) demonstrates the front view of the designed antenna, where $c$ denotes the distance between the center of the patch and the upper edge of the substrate, $e$ is distance between the feeding
TABLE 1: Design dimensions of designed antenna.

\begin{tabular}{lc}
\hline Dimension name & Dimension value \\
\hline$a$ & $10 \mathrm{~mm}$ \\
$b$ & $32 \mathrm{~mm}$ \\
$h$ & $65 \mathrm{~mm}$ \\
$c$ & $16 \mathrm{~mm}$ \\
$e$ & $9.35 \mathrm{~mm}$ \\
$l^{\prime}$ & $22.7 \mathrm{~mm}$ \\
$w$ & $22.7 \mathrm{~mm}$ \\
$h 1$ & $10.5 \mathrm{~mm}$ \\
$h 2$ & $29 \mathrm{~mm}$ \\
$h 3$ & $10 \mathrm{~mm}$ \\
$w 1$ & $2.5 \mathrm{~mm}$ \\
$w 2$ & $1.2 \mathrm{~mm}$ \\
$w 3$ & $2.5 \mathrm{~mm}$ \\
\hline
\end{tabular}

point and the patch edge, and $l^{\prime}$ and $w$ represent the physical length and physical width of patch, respectively.

Figure 1(d) illustrates the dimension of equal-split Wilkinson power divider, which is for $50 \Omega$ system impedance in working band. Width of the $50 \Omega$ transmission lines is $w 1=2.5 \mathrm{~mm}$. And the width of the quarterwavelength transmission lines is $w 2=1.2 \mathrm{~mm}$, which have a characteristic impedance of $70.7 \Omega$. The dielectric constant of the external layer substrate is 16 with its thickness being $4 \mathrm{~mm}$, while the dielectric constant of the internal layer substrate is 2.65 with its thickness being $1 \mathrm{~mm}$. Design dimensions of the proposed antenna are shown in Table 1.

\section{Analysis of the Antenna Structure}

For main $\mathrm{TM}_{01}$ mode, the $E$-field radiation is given by [17]

$$
\begin{aligned}
E_{\theta} & =j \frac{4 k_{0} U_{01}}{\lambda r} e^{-j k_{0} r} e^{j((u+v) / 2)} \sin \left(\frac{u}{2}\right) \cos \left(\frac{v}{2}\right) \\
. & {\left[\frac{w^{2}}{u^{2}}+\frac{l^{2}}{v^{2}-\pi^{2}}\right] \sin \theta \sin \varphi \cos \varphi, } \\
E_{\varphi}= & j \frac{4 k_{0} U_{01}}{\lambda r} e^{-j k_{0} r} e^{j((u+v) / 2)} \sin \left(\frac{u}{2}\right) \cos \left(\frac{v}{2}\right) \\
& {\left[\frac{w^{2} \cos ^{2} \varphi}{u^{2}}+\frac{l^{2} \sin ^{2} \varphi}{v^{2}-\pi^{2}}\right] \sin \theta \cos \theta, }
\end{aligned}
$$

where $u=k_{0} w \sin \theta \cos \varphi, v=k_{0} l \sin \theta \cos \varphi, k_{0}=2 \pi / \lambda, w$ is the effective width of the patch, $l$ is the effective length of the patch, and $U_{01}$ denotes the voltage of mode $\mathrm{TM}_{01}$ at patch corner point. 


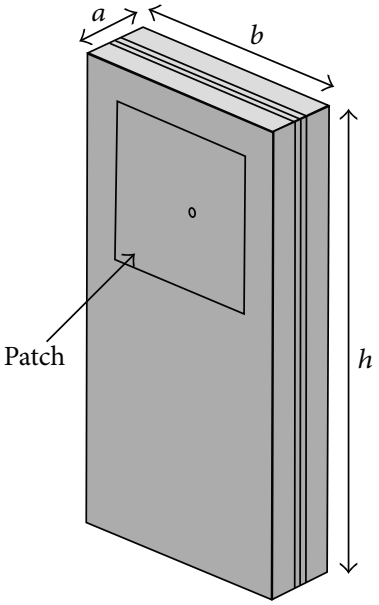

(a)

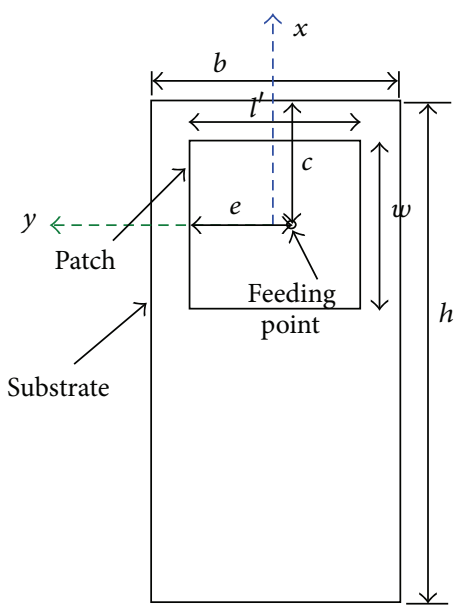

(c)

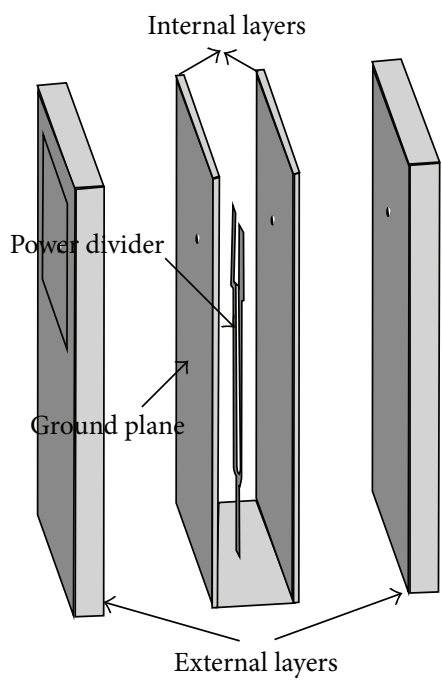

(b)

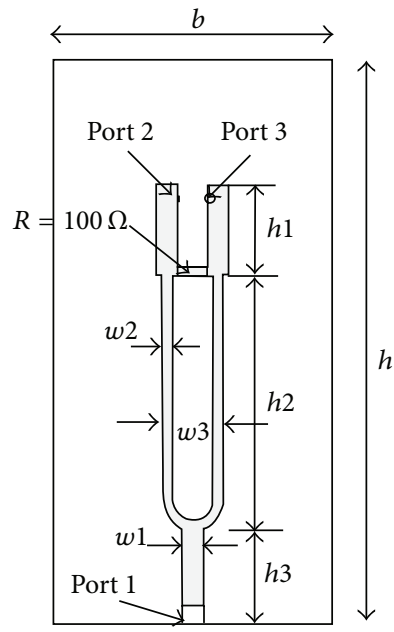

(d)

Figure 1: Model of the designed antenna.

In $E$-plane $\left(\varphi=90^{\circ}\right): u=0$ and $v=k_{0} l \sin \theta$, (1) can be simplified as

$$
\begin{aligned}
& E_{\theta}=j \frac{2 w U_{01}}{\lambda r} e^{-j k_{0} r} e^{j\left(k_{0} l / 2\right) \sin \theta} \cos \left(\frac{k_{0} l}{2} \sin \theta\right), \\
& E_{\varphi}=0 .
\end{aligned}
$$

The designed antenna under investigation can be treated as an array with two horizontally polarized microstrip antenna elements lying along the $z$-axis, as shown in Figure 2. Element 1 is placed on the positive $z$-axis, while element 2 is on the negative $z$-axis. Both of the elements are fed by two signals with the same amplitude and phase. The total field radiated by two elements, assuming no coupling and no difference in excitation between the elements, is equal to the sum of the two antenna elements. And the radiation field of element 1 in the $y$ - $z$ plane is given by

$$
E_{1 \theta}=j \frac{2 w U_{01}}{\lambda r 1} e^{-j k_{0} r 1} e^{j\left(k_{0} l / 2\right) \sin \theta} \cos \left(\frac{k_{0} l}{2} \sin \theta\right) .
$$

Since element 2 rotates $180^{\circ}$ around $x$-axis and faces negative $z$-axis compared to element 1 , far field radiation pattern of element 2 in $y$ - $z$ plane is given by

$$
\begin{aligned}
& E_{2 \theta} \\
& =j \frac{2 w U_{01}}{\lambda r 2} e^{-j k_{0} r 2} e^{j\left(k_{0} l / 2\right) \sin (\pi+\theta)} \cos \left(\frac{k_{0} l}{2} \sin (\pi+\theta)\right) .
\end{aligned}
$$

Note that element 1 and element 2 share one common ground; in this case, $d=0$ and $r 1=r 2=r$. The total field radiated by the elements is given by

$$
\begin{aligned}
E_{\theta} & =E_{1 \theta}+E_{2 \theta}=j \frac{2 w U_{01}}{\lambda r} e^{-j k_{0} r} \cos \left(\frac{k_{0} l}{2} \sin \theta\right) \\
\cdot & {\left[e^{j\left(k_{0} l / 2\right) \sin \theta}+e^{-j\left(k_{0} l / 2\right) \sin \theta}\right]=j \frac{4 w U_{01}}{\lambda r} e^{-j k_{0} r} } \\
& \cdot \cos ^{2}\left(\frac{k_{0} l}{2} \sin \theta\right) .
\end{aligned}
$$




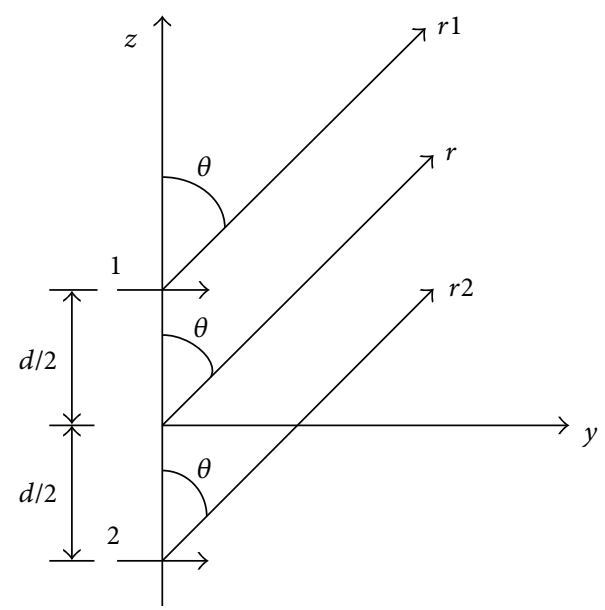

FIGURE 2: Geometry of a two-element array lying along the $z$-axis.

TABLE 2: Roundness and gain variation against physical length $l^{\prime}$.

\begin{tabular}{lccc}
\hline$g$ & Physical length $l^{\prime}(\mathrm{mm})$ & Roundness & Gain variation $(\mathrm{dBi})$ \\
\hline 2 & $\lambda / 2$ & 0 & $\infty$ \\
4 & $\lambda / 4$ & 0.25 & 6.0 \\
6 & $\lambda / 6$ & 0.56 & 2.4 \\
8 & $\lambda / 8$ & 0.73 & 1.4 \\
10 & $\lambda / 10$ & 0.82 & 0.8 \\
16 & $\lambda / 16$ & 0.93 & 0.3 \\
20 & $\lambda / 20$ & 0.95 & 0.2 \\
\hline
\end{tabular}

Since effective length $l$ is a little bit longer than physical length $l^{\prime}$ and effective length $l$ is in proportion to physical length $l^{\prime}$, it is tenable using physical length $l^{\prime}$ to replace effective length $l$ in (5). Thus for the two-element array of constant amplitude, approximate formula for directivity as a function of the directional angles in $y-z$ plane is represented by

$$
D=D_{0} \cos ^{4}\left(\frac{k_{0} l^{\prime}}{2} \sin \theta\right)
$$

where $D_{0}$ is the maximum directivity.

Suggest that physical length $l^{\prime}=\lambda / g$, where $g$ represents a parameter. Roundness of radiation pattern is $R=D_{\min } / D_{\max }$ and gain variation around the $E$-plane is $V=|10 \cdot \log R| \mathrm{dBi}$.

Table 2 shows the formula calculated roundness and gain variation against physical length $l^{\prime}$. Taking $l^{\prime}$ equal to $\lambda / 4$, for example, $D_{\min }=0.25 D_{0}$ and $D_{\max }=D_{0}$, roundness $R=$ $D_{\min } / D_{\max }=0.25$ and gain variation is $V=|10 \cdot \log (0.5)| \approx$ $6 \mathrm{dBi}$. Roundness and gain variation with some other physical length $l^{\prime}$ are calculated by using the same procedure shown in Table 2. Roundness and gain variation against physical length $l^{\prime}$ are plotted in Figure 3. It is clearly seen that as physical length $l^{\prime}$ decreases, the roundness increases and gain variation decreases.

From Table 2 and Figure 3, the following results are clear: (a) the back-to-back microstrip antenna radiates omnidirectional pattern; (b) by decreasing the physical length $l^{\prime}$,

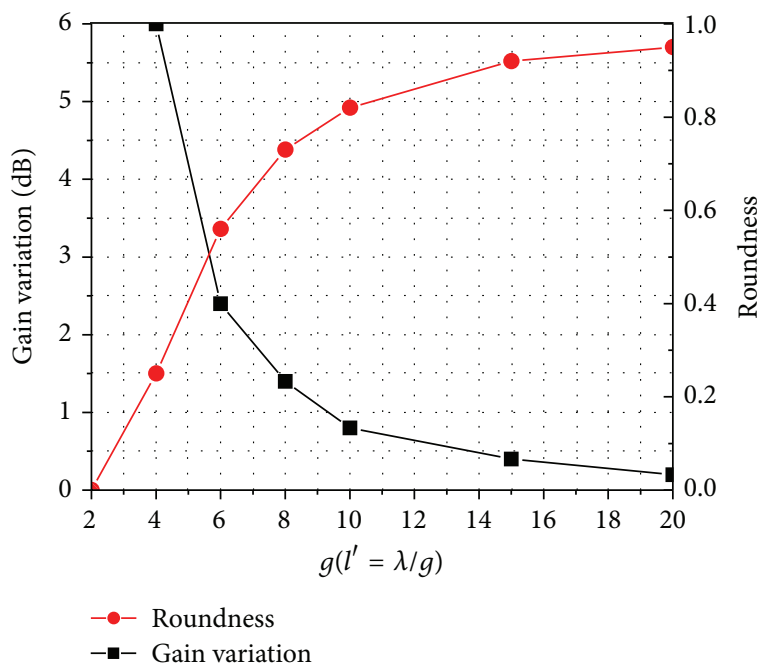

FIGURE 3: Roundness and gain variation against $l^{\prime}$.

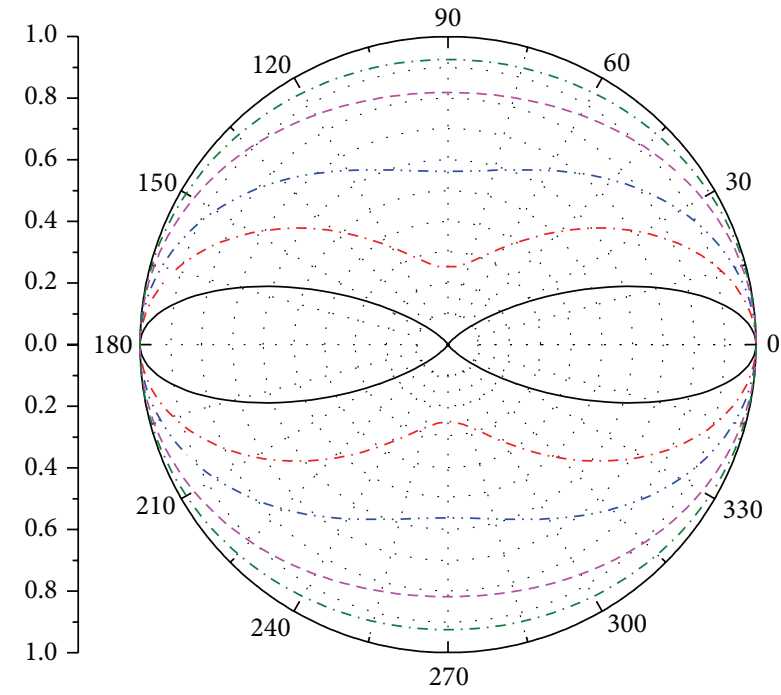

$-g=2$
$-\cdot-g=4$
$\cdots-g=6$

FIGURE 4: Formula calculated normalized radiation pattern on $y-z$ plane against $l^{\prime}$.

roundness increases and gain variation decreases. In general, physical length $l^{\prime}$ of patch is less than $\lambda / 2$, and it can be decreased by increasing dielectric constant of substrate. In this way, gain variation of the omnidirectional pattern will be decreased and better omnidirectional radiation pattern will be achieved by using higher permittivity material as shown in Figure 4. However, it is well known that such patch size reduction often brings decreased bandwidth, increased losses (lower efficiency), and matching problems, so selecting a proper substrate dielectric constant and physical length $l^{\prime}$ is quit important to achieve better antenna performance. There are three ways to increase antenna impedance bandwidth: (1) increasing substrate thickness, (2) using U-slot wideband 
TABLE 3: Comparison of gain variations.

\begin{tabular}{lcccc}
\hline & Physical length $l^{\prime}$ & & By formula $(\mathrm{dBi})$ & By HFSS $(\mathrm{dBi})$ \\
\hline Patch number 1 & $61.5 \mathrm{~mm}$ & $0.323 \lambda$ & 11.1 & 7.0 \\
Patch number 2 & $44.1 \mathrm{~mm}$ & $0.232 \lambda$ & 5 & 3.1 \\
Patch number 3 & $31.4 \mathrm{~mm}$ & $0.165 \lambda$ & 2.4 & 1.0 \\
Patch number 4 & $25.2 \mathrm{~mm}$ & $0.132 \lambda$ & 1.54 & 0.8 \\
\hline
\end{tabular}

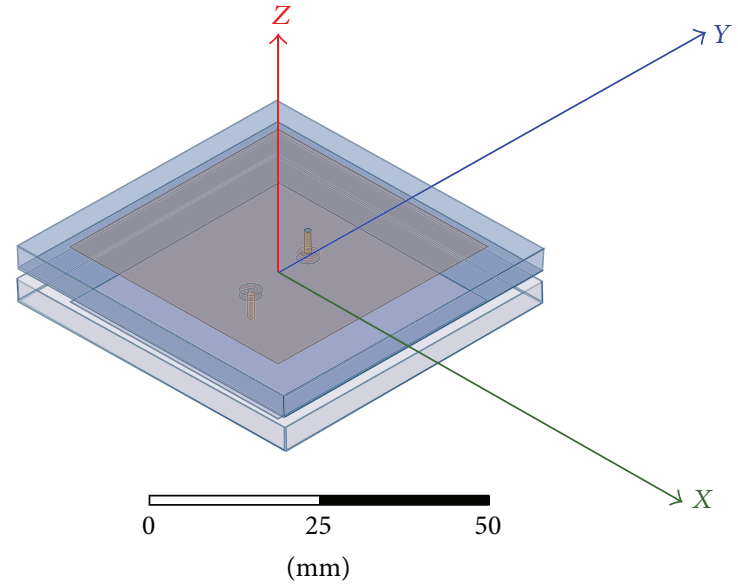

Figure 5: Model of simplified antenna structure.

patch to replace rectangular patch, and (3) using $\mathrm{H}$-shaped patch to decrease patch physical length without increasing substrate dielectric constant.

In order to verify the accuracy of formula (6) calculated directivity in $y-z$ plane of the proposed antenna, gain variation comparisons between formula calculated and HFSS simulated are made. For simplifying the simulation processing, power divider is removed from designed antenna; two patches are excited by two ports with the same amplitude and phase, as shown in Figure 5. By choosing different substrate dielectric constant, different physical length $l^{\prime}$ is got and different gain variation on main radiation plane is obtained.

There are total of four different patches whose physical length is shown in Table 3; physical length $l^{\prime} 1$ of patch number 1 is $61.5 \mathrm{~mm}$ with substrate dielectric constant $\varepsilon_{r}$, that is, $2 ; l^{\prime} 2$ of patch number 2 is $44.1 \mathrm{~mm}$ with substrate $\varepsilon_{r}$, that is, $4 ; l^{\prime} 3$ of patch number 3 is $31.4 \mathrm{~mm}$ with substrate $\varepsilon_{r}$, that is, $8 ; l^{\prime} 4$ of patch number 4 is $25.2 \mathrm{~mm}$ with substrate $\varepsilon_{r}$, that is, 12. Four antennas from number 1 to number 4 are working in the same center frequency $2.35 \mathrm{GHz}$. Normalized simulated radiation patterns (by Ansoft HFSS) in $y-z$ plane with different patches length are indicated in Figure 6. It is clearly seen that as physical length decreases, better omnidirectional performance is obtained, which has the same variation tendency as formula (6) calculated results.

Table 3 summarizes the specified numeric comparison of formula (6) calculated gain variation and HFSS simulated gain variation by given different patches physical length. Physical length $l^{\prime}$ of patch number 4 is $25.2 \mathrm{~mm}$, which is nearly $l^{\prime} \approx 0.132 \lambda$; thus $D_{\min }=1.426 D_{0}$ and $D_{\max }=D_{0}$,

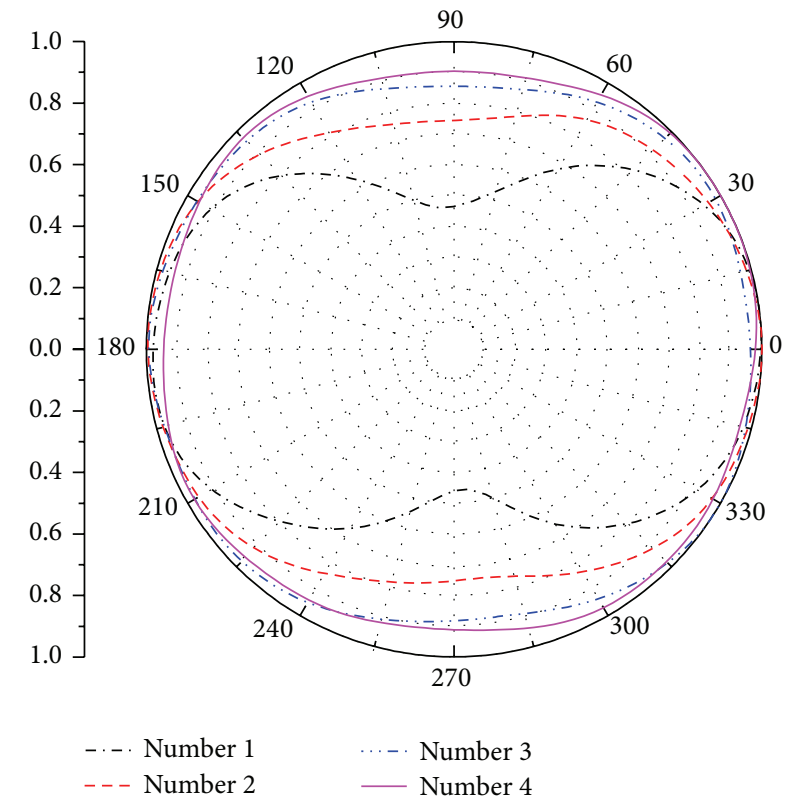

FIGURE 6: HFSS simulated normalized radiation pattern in $y$ - $z$ plane.

so roundness $R=D_{\min } / D_{\max }=1.426$ and calculated gain variation is $V=|10 \cdot \log (0.078)| \approx 1.54 \mathrm{dBi}$. By using the same procedure, gain variation of other patches physical length can be calculated as shown in Table 3 .

Gain variation difference between formula calculated and HFSS simulated results is shown in Figure 6. Gain variation difference of patch number 1 is $4.1 \mathrm{dBi}$ and those of patch number 2 , patch number 3 , and patch number 4 are $1.9 \mathrm{dBi}$, $1.4 \mathrm{dBi}$, and $0.74 \mathrm{dBi}$, respectively (Figure 7). As physical length is getting smaller, less difference between formula calculated result and HFSS simulated result is achieved. Both results show that better gain variation performance is obtained as patch physical length is getting shorter.

\section{Simulated and Measured Results}

In this section, several numerical simulations are conducted using Ansoft HFSS to validate the performance of the proposed algorithm. Better omnidirectional performance of this antenna can be obtained by increasing substrate dielectric constant to reduce patch physical length. However, such patch length reduction often brings decreased bandwidth, increased losses (lower efficiency), and matching problems. 


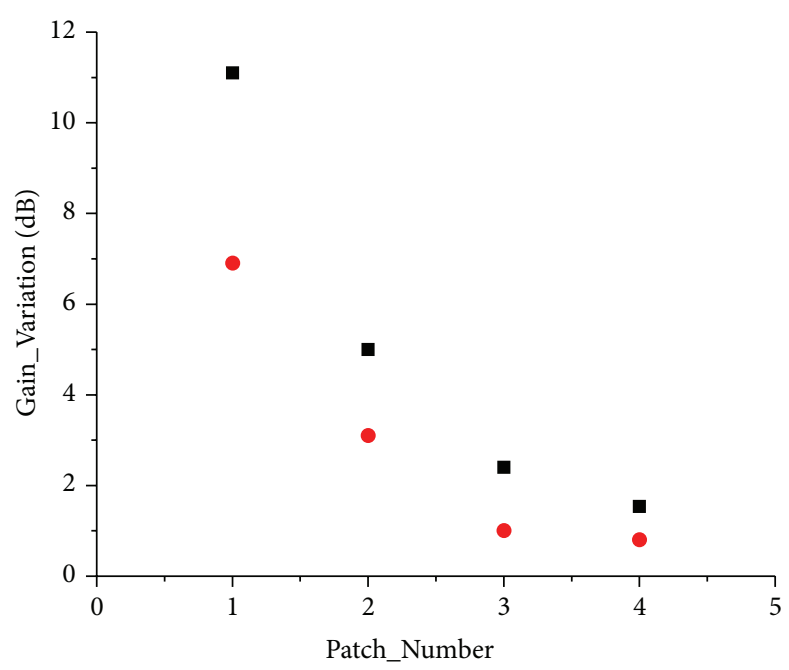

By formula

- By HFSS

FIgURE 7: Gain variation difference in $y$ - $z$ plane.

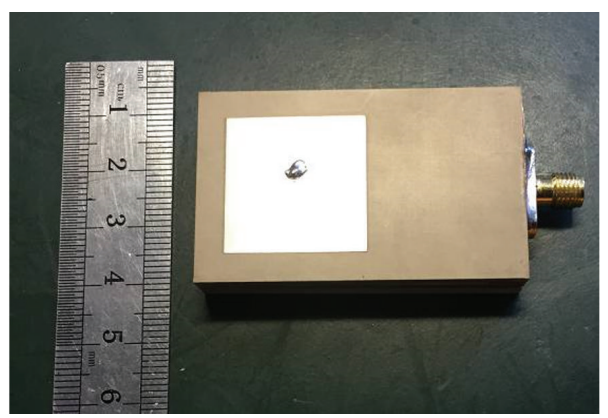

FIGURE 8: Designed antenna.

Therefore, we set the substrate dielectric constant as $\varepsilon_{r}=$ 16 to optimize antenna performance. And the optimized parameters of the antenna are as follows: $a=6 \mathrm{~mm}, b=$ $32 \mathrm{~mm}, c=16 \mathrm{~mm}, e=9.35 \mathrm{~mm}, w=22.1 \mathrm{~mm}, l^{\prime}=22.1 \mathrm{~mm}$, $h=65 \mathrm{~mm}, h 1=10.5 \mathrm{~mm}, h 2=29 \mathrm{~mm}, h 3=10 \mathrm{~mm}, w 1=$ $2.5 \mathrm{~mm}, w 2=1.2 \mathrm{~mm}$, and $w 3=2.5 \mathrm{~mm}$.

Figure 8 illustrates the realization of the designed horizontally polarized omnidirectional microstrip antenna with a SMA connector. Both the simulated and measured values of the designed antenna's $S_{11}$ parameter are shown in Figure 9. It is clear that the band-width of the antenna is about $1.6 \%$ (from $1.562 \mathrm{GHz}$ to $1.587 \mathrm{GHz}$ ) with center frequency $1.575 \mathrm{GHz}$, and measured result agrees well with the simulated one. Note that bandwidth of designed antenna is narrow; one feasible way to overcome narrow bandwidth performance is to use wideband U-slot patch to replace square patch.

Simulated three-dimensional radiation pattern of the designed antenna is shown in Figure 10. Peak gain of the designed antenna is about $2.2 \mathrm{dBi}$. It is obvious that the radiation pattern is omnidirectional in $y-z$ plane, and the gain variation in $y-z$ plane is less than $\pm 0.4 \mathrm{dBi}$.

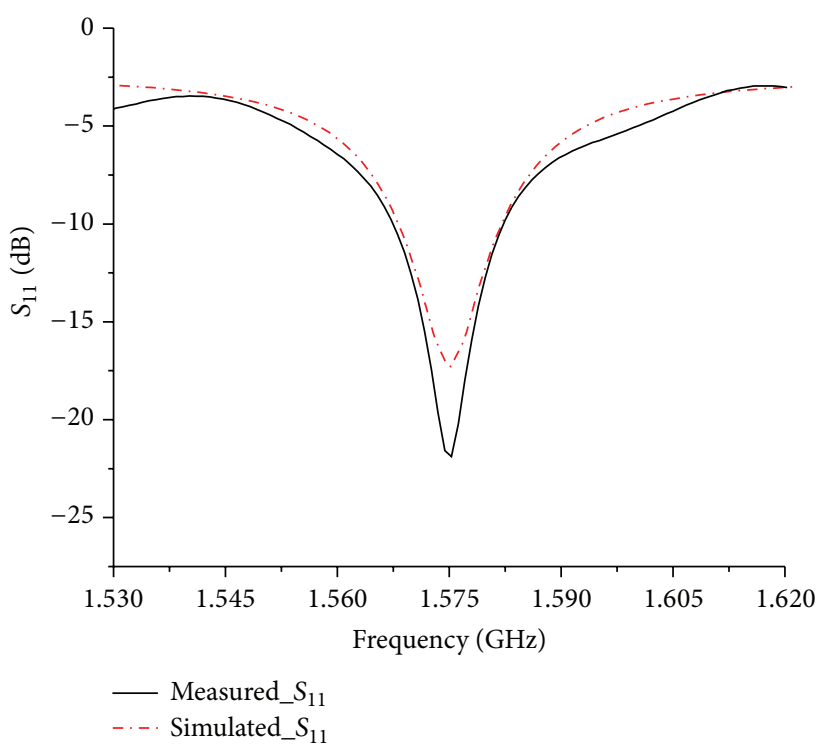

FiguRE 9: Simulated and measured $S_{11}$. 


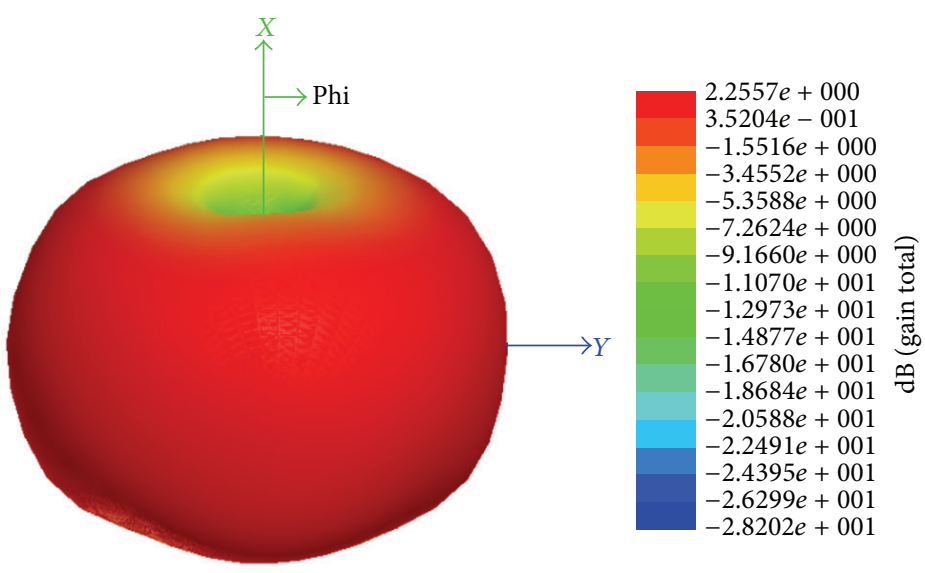

FIGURE 10: Three-dimensional radiation pattern.

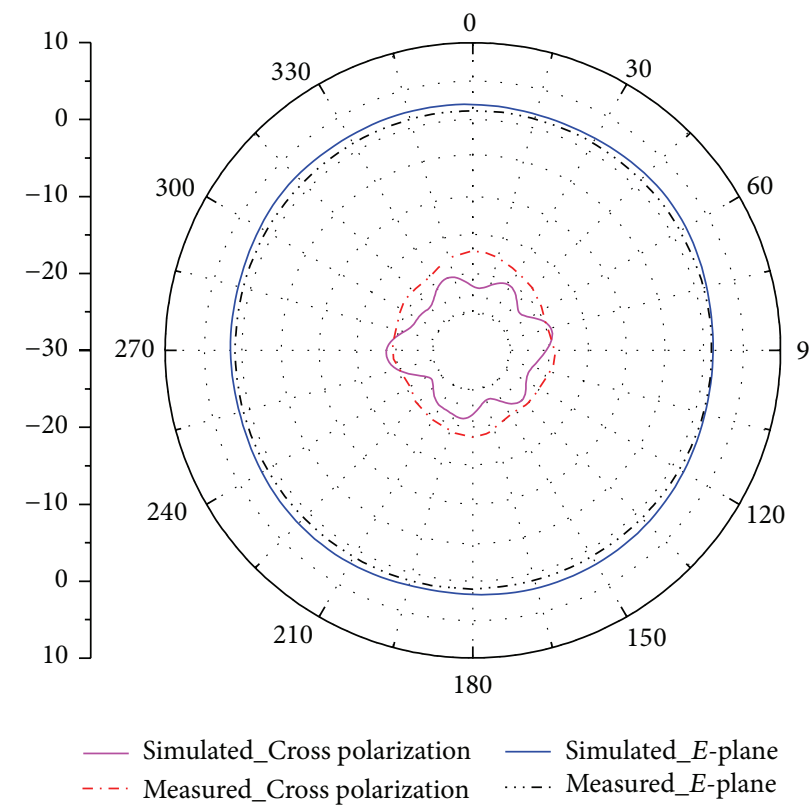

(a)

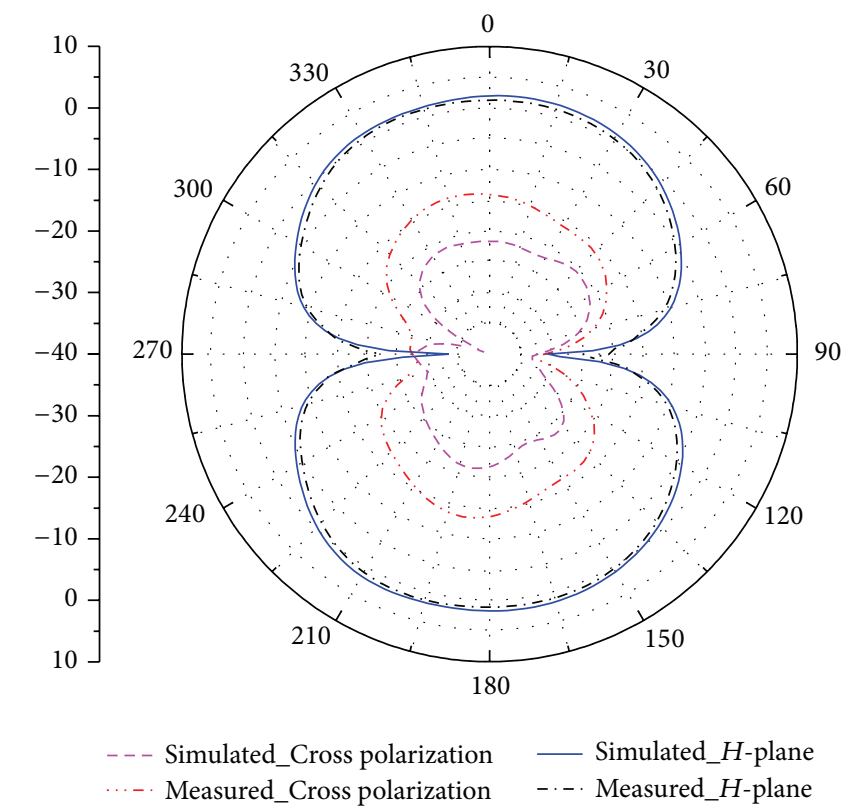

(b)

FIGURE 11: Simulated and measured radiation pattern. (a) E-plane. (b) $H$-plane.

Figures 11(a) and 11(b) depict the simulated and measured main polarization pattern and cross polarization pattern in $E$-plane and $H$-plane, respectively. Peak gain of the designed antenna is about $2.2 \mathrm{dBi}$; antenna efficiency is higher than $90 \%$ in the operating band. According to the simulation results, the gain variation in $E$-plane pattern is about $0.58 \mathrm{dBi}$, while the measured gain variation is about $0.8 \mathrm{dBi}$ and cross polarization level is $-20 \mathrm{dBi}$ lower than that of main polarization.

Figure 12 shows the simulated radiation patterns of designed antenna in this paper and dipole antenna in $E$-plane and $H$-plane. Both designed antenna and dipole antenna can obtain omnidirectional radiation pattern. Peak gains of those two antennas are almost the same, but antenna in this paper is with better omnidirectional performance.
Moreover, designed antenna is horizontally polarized rather than vertically polarized.

\section{Conclusion}

A new horizontally polarized omnidirectional microstrip antenna is presented in this paper. Formula of directivity as a function of the directional angles in main lobe radiation plane of designed antenna is deduced. Better omnidirectional characteristic of this antenna can be obtained by increasing substrate dielectric constant or decreasing patch physical length. Both simulated and measured experiments are carried out to verify the performance of the proposed antenna architecture. It has been shown that the peak gain is about $2.2 \mathrm{dBi}$, the antenna efficiency is higher than $90 \%$ in operating band, 


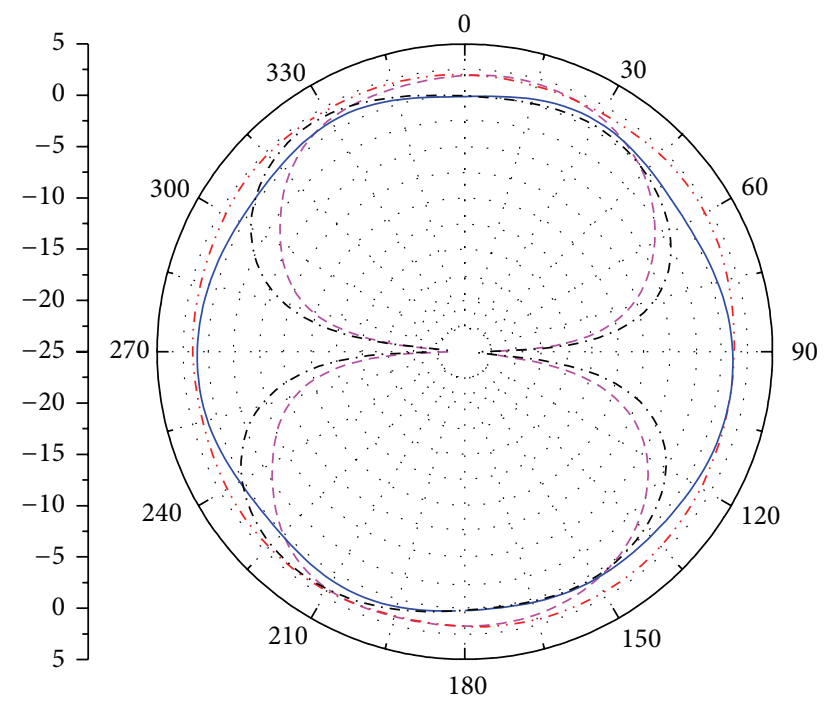

-- Designed antenna_H-plane $\quad-$ Dipole antenna_H-plane
... - Designed antenna_E-plane $\quad-$. Dipole antenna_E-plane

FIGURE 12: Simulated radiation patterns of designed antenna and dipole antenna.

and the cross polarization level is $-20 \mathrm{dBi}$ lower than that of main polarization. Moreover, the $E$-plane gain variation of the designed antenna is less than $\pm 0.4 \mathrm{dBi}$ which indicates a satisfactory omnidirectional radiation pattern.

\section{Conflict of Interests}

The authors declare that there is no conflict of interests regarding the publication of this paper.

\section{Acknowledgments}

This work was supported in part by the National Natural Science Foundation of China (no. 61271416, no. 61301093, and no. 61501376), the Fundamental Research Funds for the Central Universities (no. 3102014KYJD027), and NPU Foundation for Fundamental Research (no. JCY20130132).

\section{References}

[1] A. Chen, T. Jiang, Z. Chen, and D. Su, "A novel low-profile wideband UHF antenna," Progress in Electromagnetics Research, vol. 121, pp. 75-88, 2011.

[2] T. J. Judasz and B. B. Balsley, "Improved theoretical and experimental models for the coaxial colinear antenna," IEEE Transactions on Antennas and Propagation, vol. 37, no. 3, pp. 289-296, 1989.

[3] M. Sierra-Pérez, F. L. Heras-Andrés, and J. A. G. de Lope, "Low-cost printed collinear array antenna," IEEE Antennas and Propagation Magazine, vol. 43, no. 5, pp. 23-30, 2001.

[4] H.-Y. Xu, H. Zhang, K. Lu, and X.-F. Zeng, "A holly-leafshaped monopole antenna with low RCS for UWB application," Progress in Electromagnetics Research, vol. 117, pp. 35-50, 2011.
[5] P. Li, K. M. Luk, and K. L. Lau, "An omnidirectional high gain microstrip antenna array mounted on a circular cylinder," in Proceedings of the IEEE Antennas and Propagation Society International Symposium, vol. 4, pp. 698-701, Columbus, Ohio, USA, June 2003.

[6] A. Narbudowicz, X. Bao, M. Ammann, H. Shakhtour, and D. Heberling, "Circularly polarized antenna with steerable dipolelike radiation pattern," IEEE Transactions on Antennas and Propagation, vol. 62, no. 2, pp. 519-526, 2014.

[7] H. Iwasaki, "A back-to-back rectangular-patch antenna fed by a CPW,' IEEE Transactions on Antennas and Propagation, vol. 46, no. 10, pp. 1527-1530, 1998.

[8] D. I. Wu, "Omnidirectional circularly-polarized conformal microstrip array for telemetry applications," in Proceedings of the Antennas and Propagation Society International Symposium, vol. 2, pp. 998-1001, Newport Beach, Calif, USA, June 1995.

[9] K. Wei, J.-Y. Li, L. Wang, and Z. Xing, "A new omnidirectional circular polarization microstip antenna," Progress in Electromagnetics Research Letters, vol. 53, pp. 45-50, 2015.

[10] S. Wang and H. Arai, "A horizontally polarized notch array antenna with good radiation identity in horizontal plane and its characteristic modes analysis," in Proceedings of the IEEE Antennas and Propagation Society International Symposium, pp. 1073-1074, IEEE, Memphis, Tenn, USA, July 2014.

[11] X. L. Quan, R. L. Li, J. Y. Wang, and Y. H. Cui, “Development of a broadband horizontally polarized omnidirectional planar antenna and its array for base stations," Progress in Electromagnetics Research, vol. 128, pp. 441-456, 2012.

[12] C.-C. Lin, L.-C. Kuo, and H.-R. Chuang, "A horizontally polarized omnidirectional printed antenna for WLAN applications," IEEE Transactions on Antennas and Propagation, vol. 54, no. 11, pp. 3551-3556, 2006.

[13] Y. Li, Z. Zhang, and Z. Feng, "A leaky wave slot antenna array using single metal layer with azimuthally omnidirectional pattern," Progress in Electromagnetics Research, vol. 140, pp. 199212, 2013.

[14] X. Chen, K. Huang, and X.-B. Xu, "A novel planar slot array antenna with omnidirectional pattern," IEEE Transactions on Antennas and Propagation, vol. 59, no. 12, pp. 4853-4857, 2011.

[15] Y. Li, Z. Zhang, Z. Feng, and M. F. Iskander, "Design of pentaband omnidirectional slot antenna with slender columnar structure," IEEE Transactions on Antennas and Propagation, vol. 62, no. 2, pp. 594-601, 2014.

[16] A. Y. Svezhentsev, P. J. Soh, S. Yan, V. Volskiy, and G. A. E. Vandenbosch, "Omnidirectional horizontally-polarized cylindrical microstrip antenna with two parasitic patches," in Proceedings of the IEEE Antennas and Propagation Society International Symposium (APSURSI '14), pp. 1863-1864, IEEE, Memphis, Tenn, USA, July 2014.

[17] S. S. Zhong, "Slot antenna and microstrip antenna," in Proceedings of the 8th International Conference on Antenna Theory and Techniques (ICATT '11), Section 1, pp. 272-274, Beijing, China, September 2011. 


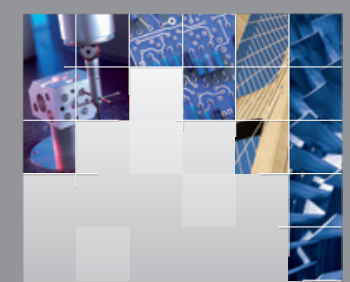

\section{Enfincering}
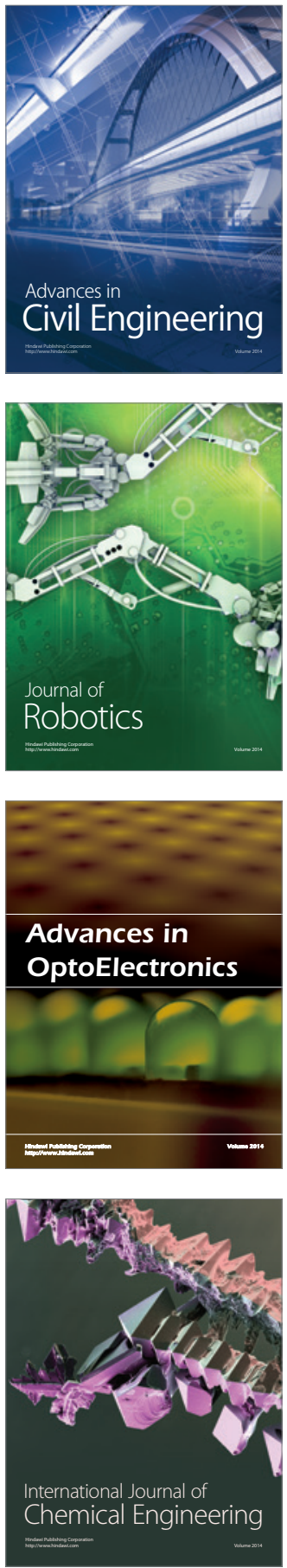

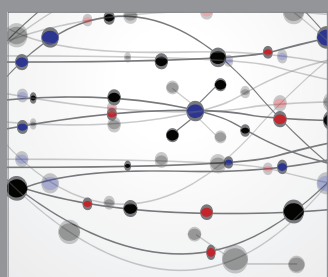

The Scientific World Journal

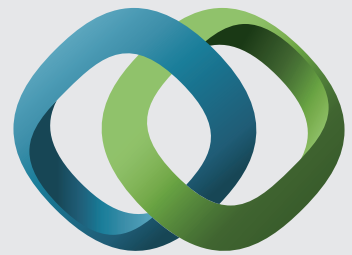

\section{Hindawi}

Submit your manuscripts at

http://www.hindawi.com
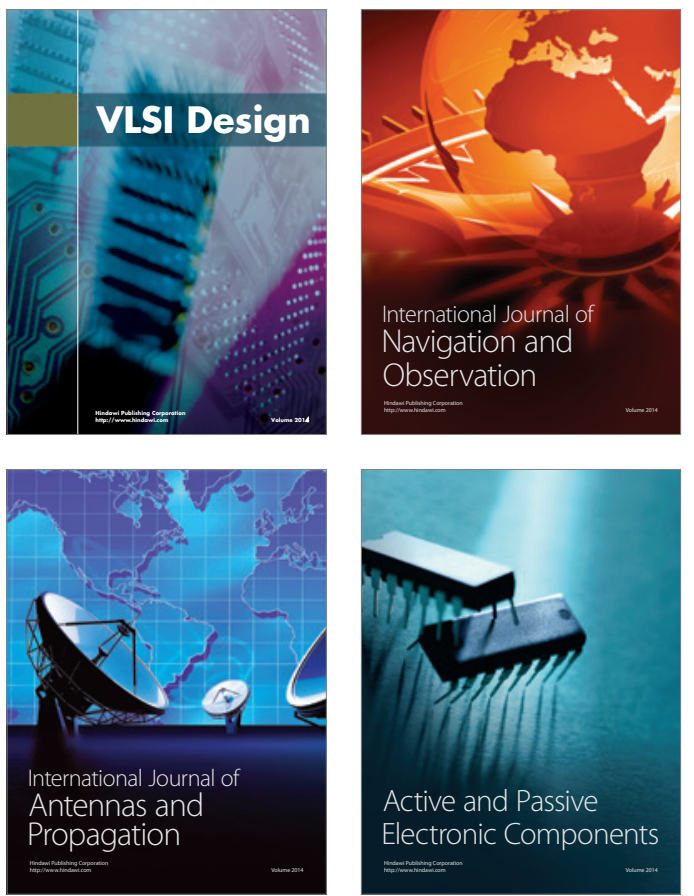
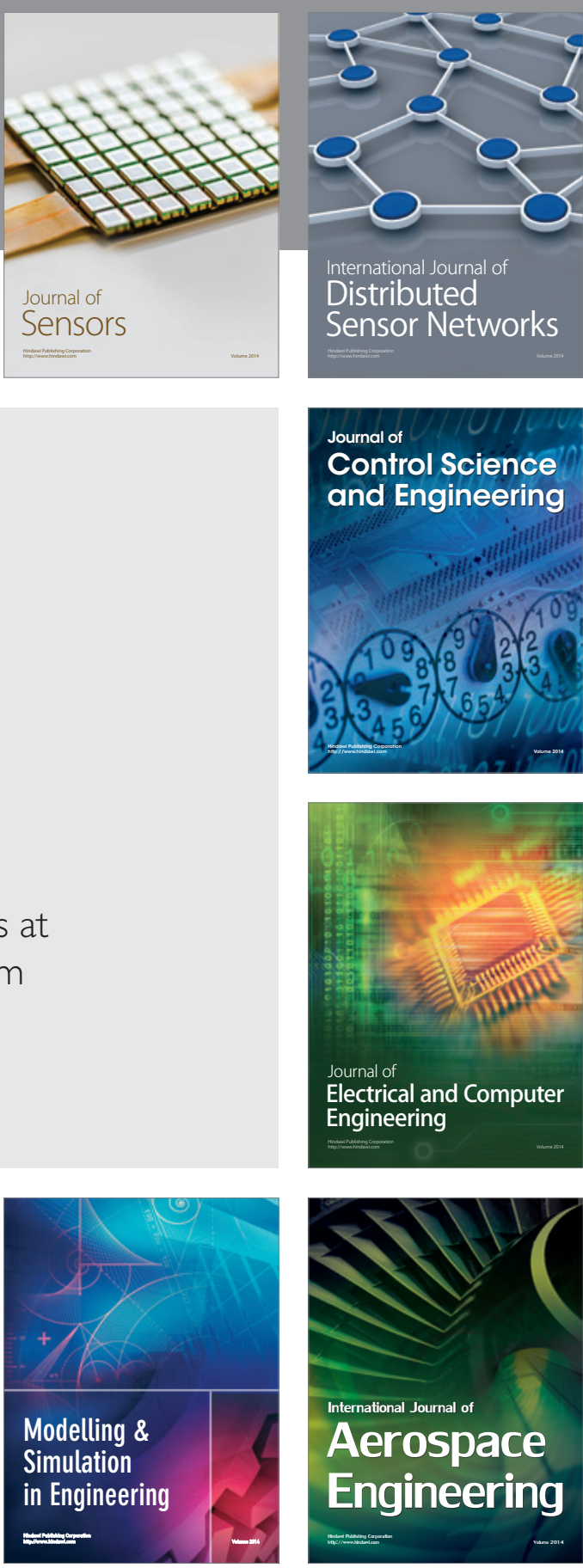

International Journal of

Distributed

Sensor Networks

Journal of

Control Science

and Engineering
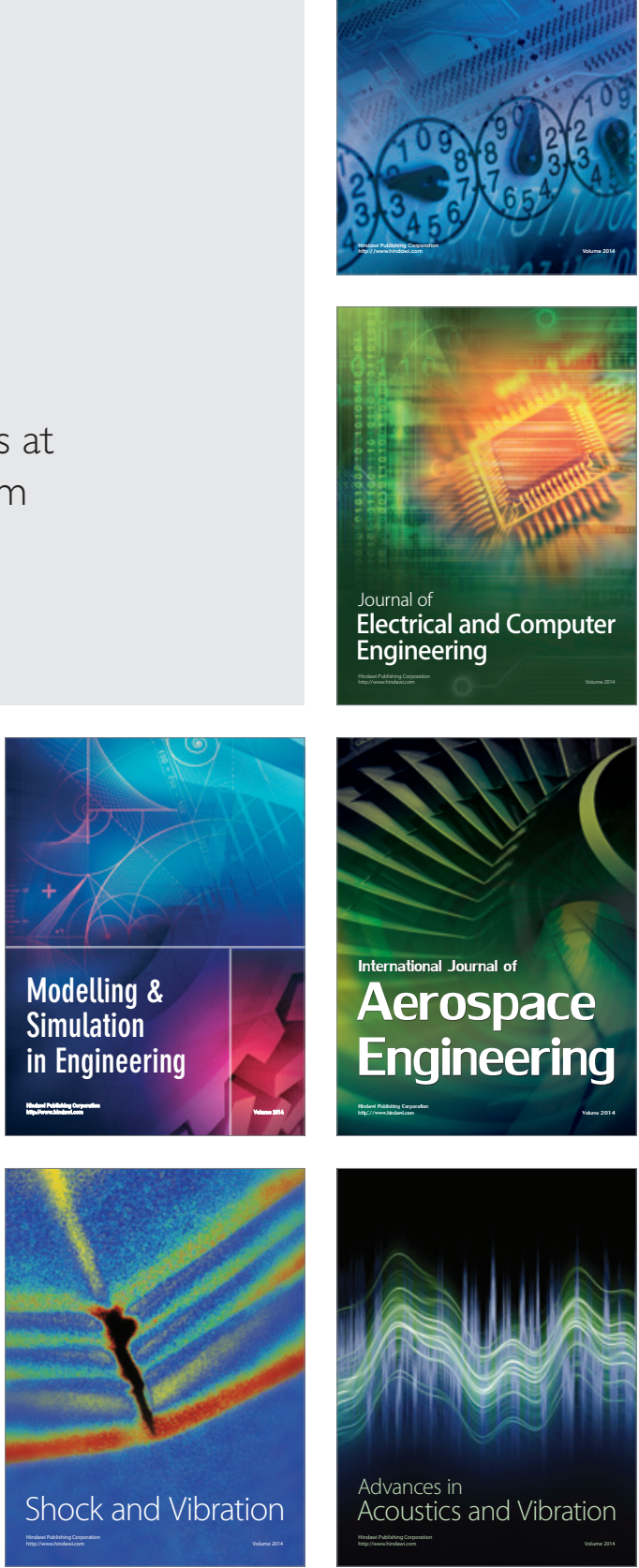